\title{
Enhanced IoT Based Child Missing Alert System Using RSSI
}

\author{
Simran Kaur Gill ${ }^{\text {a, }}$, and M. Safa ${ }^{\text {a }}$ \\ ${ }^{a}$ Dept of Information Technology, School of Computing, SRM Institute of Science \\ and Technology, Chennai, TamilNadu, India
}

\begin{abstract}
Our proposed system discusses the concept of a smart wearable device connected to their parent's mobile phone for children and their parents respectively. In this project we propose that to let the system be divided into three parts, namely the safe, intermediate and danger zones. If the child is within the safe zone, then no buzzer is sounded whereas if the child is in the intermediate range a buzzer alert will be sounded. If the child crosses the 'danger' zone, the buzzer is sounded with an immediate notification sent to the parent. In case the child goes out of danger zone, a GPS module is attached that would help parent know the exact location of the child once he/she is outside the 100 meters of radius from the parent. This project also has features to sense the child's temperature and heartbeat along with notifying the child's parent in case the child has an accident using the temperature, heartbeat and pressure sensors respectively. The RSSI is used for distance sensing whereas GSM is used for notification sending to the parent's mobile phone.
\end{abstract}

Keywords. Free Sensing, Tracking, Radio Signal Strength Information (RSSI), Global System for mobile Communications (GSM), Global Positioning System (GPS).

\section{Introduction}

We often come across billboards filled with their faces smiling at us. In most cases, these children are found safely by the government officials, while the others are lost forever. In such cases we imagine such reunions filled of tearful happiness with hugs and kisses. But fail to notice that while these children and their families, where they have found each other had a happy ending. The odd senses we feel of what they might have went through bring a chill to my spine. On occasions where we do find them, some of them are found missing a part of their body. Thus, a proper child location tracking is necessary. Therefore, we would like to propose an enhanced RSSI based Child Tracking System using IoT of independent and efficient tracking of the child while the child is nearby to the parent and aided with a GPS module when the child is far away from the reach of parents, preferably outdoors in a crowded place.

\footnotetext{
${ }^{1}$ Simran Kaur Gil,Dept of Information Technology, School of Computing, SRM Institute of Science and Technology, Chennai, Email: ss8723@srmist.edu.in.
} 


\section{Proposed System}

In our enhanced RSSI system we propose that to let the system be split into three parts, the safe, intermediate and danger zones. If the child is within the safe zone, then no buzzer is sounded whereas if the child is in the intermediate range a buzzer alert will be sounded. If the child crosses the 'danger' zone, the buzzer is sounded with an immediate notification sent to the parent. In case the child goes out of danger zone, a GPS module is attached that would help parent know the exact location of the child once he/she is outside the 100meters of radius from the parent. The RSSI is used for distance sensing whereas GSM is used for sending notification to the parent's mobile phone. The system uses the Aurdino Uno microcontroller and takes the input from the two sources. One of which is the environmental parameters Heartbeat, Temperature and Pressure Sensors. The second takes the received signal of the child from the parent using the RSSI module. And then the data is sensed in the form of dbms to check whether the child is in range of the parent mobile hotspot or not, that is 100 meters, to send the precise reading in case the child is going far away from the central point. Meanwhile using the DHT11, transducer and heart rate sensors to sense the body temperature of child along with his/her heart rate and in case if the child has fallen down, it sends the appropriate notification via sms to the parent device. Meanwhile a buzzer goes 'off' to notify the people around the child of the same situation. For a checking the precise location of the child once he/she is out of the 100 metres radius, a GPS module is also installed. The location of the child through which would be sent as a link to the parent via sms. For the purpose of sending the messages to the parent, GSM or the Global System for Mobile Communications is used.

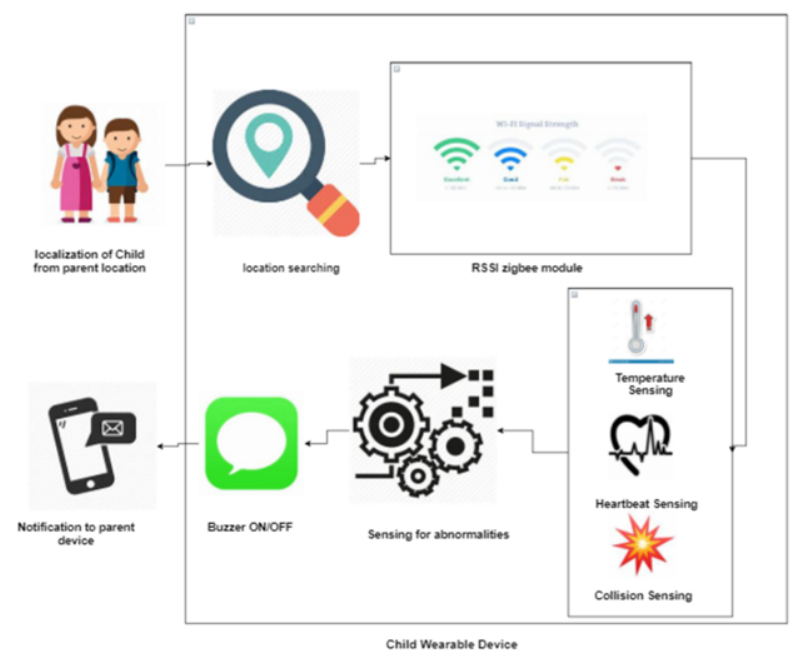

Figure 1. work flow diagram 


\section{Project Dataflow and Architecture}

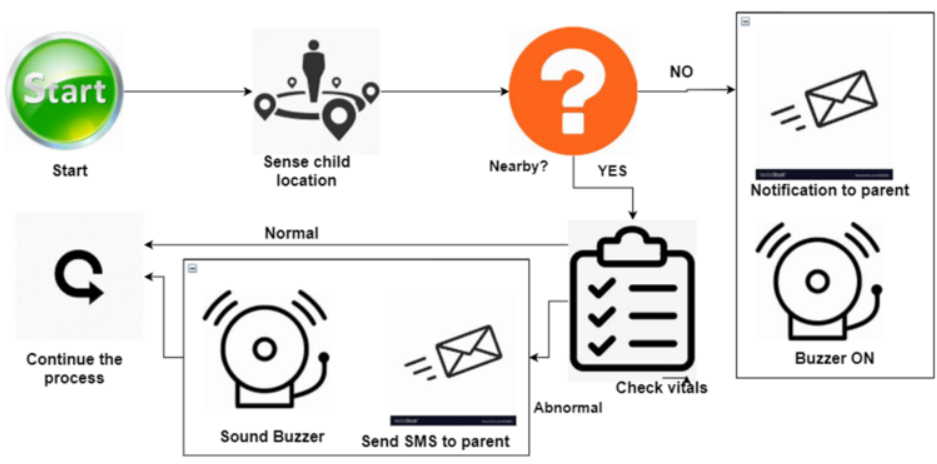

Figure 2 System Data Flow Diagram

This system consists of two separate pieces. A child wearable device and the parent mobile phone. The child wearable device consists of all the sensors, microcontroller, GPS Module, GSM, a Buzzer, an LCD, LEDs and the RSSI module. On the parent mobile phone, the wifi hotspot is used as the centre within which the RSSI senses the child location, stepping out of which a buzzer is sounded. The hardware first senses the current real time location of the child. If the child is right beside the parent, a green light is turned on whereas if the child is a little further away from the parent the yellow light is turned on else if the child goes out of the 100 meters radius, the red LED is turned on with a notification sent about the same to the parent and Buzzer is turned ON. Next, if the child has high temperature, heartbeat or if he/she fell down, an appropriate message is sent to the parent device and simultaneously a buzzer is turned ON. The LCD setup on the child wearable device also displays the same message on it. The whole process plays continuously on a loop to sense the abnormalities and report them accordingly.

\section{Implementation}

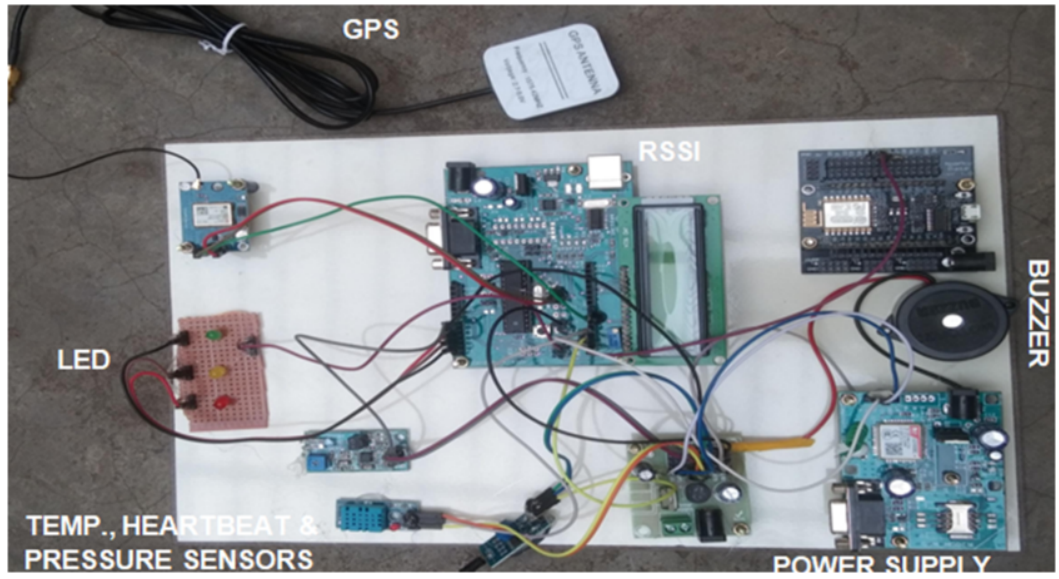

Figure 3. System Architecture 
The microcontroller contains one or more Central Processing Units (CPU). It is the unit that undertakes the administration of the various input and the output constraints. Arduino code is launched on the microcontroller. It processes the input and sends it to another module and also to control the output devices i.e., the buzzer and LCD on the child wearable device along with the LEDs and Parent notification. It accepts the temperature, heartbeat and the intensity of pressure faced by the child wearable device as the input constraints from the DHT11 and transducer sensors. In the next step, the source code is launched on the microcontroller. From here, the microcontroller begins to take the input constraints and forwards it to the next module. DHT11 sensor is used to measure the temperature and heartbeat at the moment, which is used as a constraint to decide the type and nature of the notification and whether or not to send it.

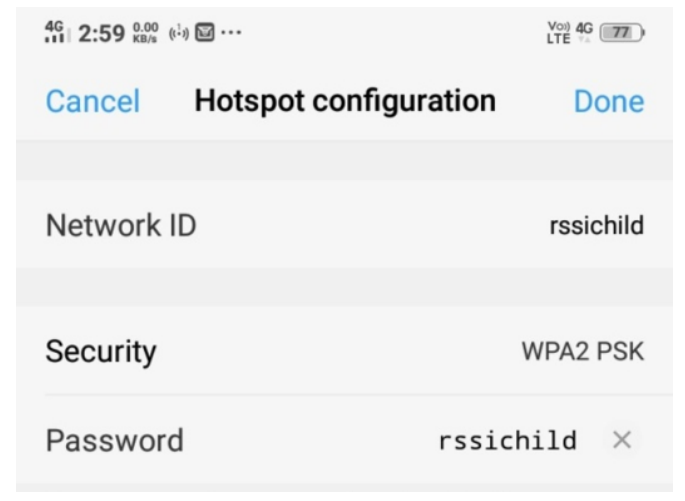

Figure 4. Wifi Configuration

The child device and the parent device are connected to each other via the parent mobile hotspot. The COM 19 available on the Aurdino Uno is used is used to sense the wifi hotspot which in turn connects and initiates localization via RSSI which then uses the parent's mobile phone as the central point to which the child's distance is calculated.A GPS module is setup on the child wearable. This is done because the Received Signal Strength Indicator only indicates the relative distance between the child and the parent. In the GPS Output, the message is made up of three major components. This message is known as the navigation message. Thus it is important to extract the specific longitude and latitude values.

\section{Sending message alert to parent mobile phone via GSM}

The proposed framework contains a set of notifications to be sent to the parent mobile, such as the child 'fell down' or 'irregular heartbeat and temperature', or even in case the child is 'out of range' when the child crosses the 100metres radius distance from the parent location. The code snippet is necessary to transfer the notification to the registered parent contact details. The notification is sent by inserting the parent's contact number in the 'phno' area whereas the ' +91 ' indicates the country code. This overall notification or alert sending work is done via the GSM module. 


\section{Input/output Result}

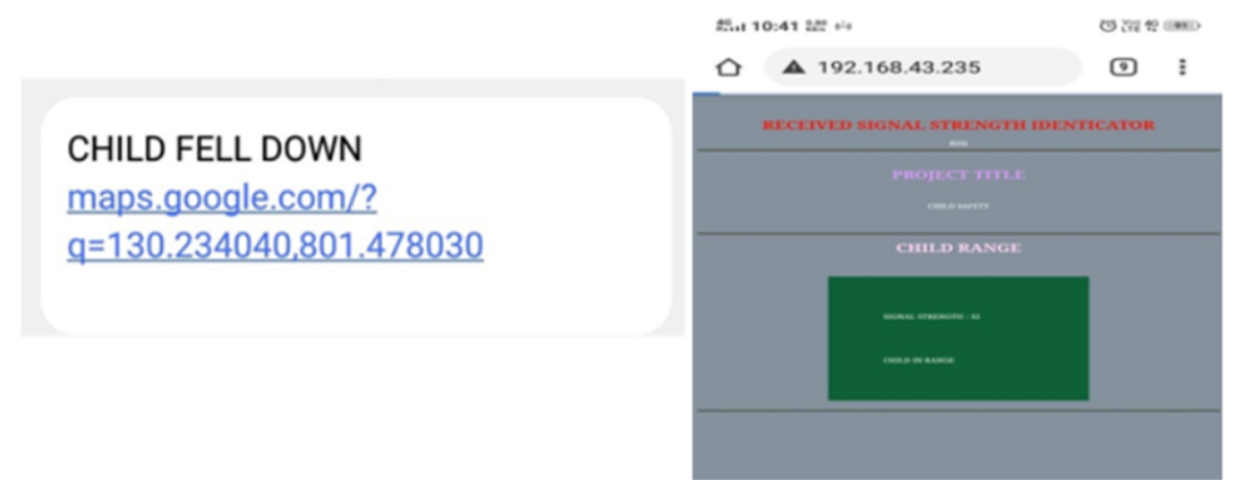

Figure 5. a). Notification with location of child and b). RSSI based Localization output for parent

\section{Conclusion}

Hence, the most useful part of this system over the other alternatives is that it can be used in any smart phone irrespective of its cost. Moreover, this system doesn't require the parents to be of any technical background. Hence can easily be used by every parent, unbeknownst of their study background. The aim of this project is to aid the parents pinpoint their child's location and detect when he/she is fallen astray, in the most unchallenging way. This device can help the government officials by pointing out all the possible hotspots from which the children are abducted with the timings around which these cases frequently take place. With the hotspots and timings noted, more attention and security during this time range when these cases occur, this project can help lessen the cases of child missing and kidnappings. On the other hand, The Device can fail to prove to accuracy during really high temperatures, as during such weathers the body temperature is significantly higher and lower during winters.

\section{References}

[1] Daniel Konings, Fakhrul Alam, Frazer Noble, and Edmund M-K Lai (2019). "Device-free Localization Systems Utilizing Wireless RSSI: A Comparative Practical Investigation".

[2] Kishore Kumar Reddy. N.G, Ramakrishnan.G and Rajeshwari.K (2017). "Ensuring Fishermen Safety through a Range Based System by Trizonal Localization using Low Power RSSI"

[3] Pradipta Ghosh, Jason A. Tran, and Bhaskar Krishnamachari (2019). "ARREST: A RSSI Based Approach for Mobile Sensing and Tracking of a Moving Object"

[4] Y. Guo, K. Huang, N. Jiang, X. Guo, Y. Li, and G. Wang, "An exponential-rayleigh model for rssbased device-free localization and tracking," IEEE Transactions on Mobile Computing, vol. 14, no. 3, pp. 484-494, 2015.

[5] D. Konings, N. Faulkner, F. Alam, F. Noble, and E. M. K. Lai, "The effects of interference on the rssi values of a zigbee based indoor localization system," in 2017 24th International Conference on Mechatronics and Machine Vision in Practice (M2VIP), 2017, Conference Proceedings, pp. 1-5.

[6] L. Oliveira, H. Li, L. Almeida, and T. E. Abrudan (2014). "Rssi-based relative localisation for mobile robots,".

[7] D. Konings, N. Faulkner, F. Alam, F. Noble, and E. M-K Lai, "Do rssi values reliably map to rss in a localization system?” in Workshop on Recent Trends in Telecommunications Research (RTTR). IEEE, 2017 
[8] I. 18305:2016, Information technology - "Real time locating systems - Test and evaluation of localization and tracking systems", ser. Standard ISO/IEC 18305:2016. ISO/IEC JT, 2016. [Online]. Available: https://www.iso.org/standard/62090.html

[9] D. Vasisht, S. Kumar, and D. Katabi (2016). "Decimeter-level localization with a single wifi access point,",

[10] Simo Särkkä, Ville V. Viikari, Miika Huusko, and Kaarle Jaakkola (2012). "Phase-Based UHF RFID Tracking With Nonlinear Kalman Filtering and Smoothing"

[11] Emidio DiGiampaolo and Francesco Martinelli (2014). "Mobile Robot Localization Using the Phase of Passive UHF RFID Signals"

[12] Mauro Boccadoro, Francesco Martinelli and Stefano Pagnottelli (2010). "Constrained and quantized Kalman filtering for an RFID robot localization problem"

[13] Ricardo Tesoriero, José A. Gallud, María D. Lozano, Víctor M. R. Penichet (2009). "Tracking Autonomous Entities using RFID Technology"

[14] SIMON J. JULIER AND JEFFREY K. UHLMANN (2004). "Unscented Filtering and Nonlinear Estimation"

[15] Piyush Raj, M. Safa, Nitish Kumar, P J Sahrudh, Shivaditya Singh. (2020). Enhanced Smart Music Controller by Applying CNN in IoT. International Journal of Advanced Science and Technology, 29(06), 2739 - 2749.

[16] Aditya Vadagave, M.Safa, Sanjit Singh Sokhi, Bhaawan Mathur, Bhagavatula Anirudh. (2020). Enhancing Smart Precision Agriculture in IoT Using Agro ecologicali Analysis. International Journal of Advanced Science and Technology, 29(06), 2750 - 2760.

[17] Abhirup Bose, M. Safa, Sanjay Bhargav Siddi, Manas Raj Anand, Vivek Kumar. (2020). Implementation of Dynamic Lighting \& Augmented Reality (DLAR) Smart Home System for Deaf and Hard-of-Hearing (DHH) Residents. International Journal of Advanced Science and Technology, 29(06), $2724-2738$.

[18] Aadhineni Ganesh, M. Safa, M.Roopanjali, P.V.K Sai Sri Harsha, D. Anjana Tulasi. (2020). An Approach for Monitoring and Analyzing Parking Occupancies using IoT. International Journal of Advanced Science and Technology, 29(06), 3536 - 3545. 